\title{
Irrigation water allocation optimization using multi-objective evolutionary algorithm (MOEA) - a review
}

\author{
Ibrahim Mwita Fanuel ${ }^{1, *}$, Allen Mushi ${ }^{2}$, and Damian Kajunguri ${ }^{1}$ \\ ${ }^{1}$ Department of Applied Mathematics and Computational Sciences, Nelson Mandela African Institution of Science Technology, \\ P.O. Box 447, Arusha, Tanzania \\ 2 Department of Mathematics, College of Natural and Applied Science, University of Dar es Salaam, P.O. Box 35062, \\ Dar es Salaam, Tanzania
}

Received: 14 April 2017 / Accepted: 10 January 2018

\begin{abstract}
This paper analyzes more than 40 papers with a restricted area of application of Multi-Objective Genetic Algorithm, Non-Dominated Sorting Genetic Algorithm-II and Multi-Objective Differential Evolution (MODE) to solve the multi-objective problem in agricultural water management. The paper focused on different application aspects which include water allocation, irrigation planning, crop pattern and allocation of available land. The performance and results of these techniques are discussed. The review finds that there is a potential to use MODE to analyzed the multi-objective problem, the application is more significance due to its advantage of being simple and powerful technique than any Evolutionary Algorithm. The paper concludes with the hopeful new trend of research that demand effective use of MODE; inclusion of benefits derived from farm byproducts and production costs into the model.
\end{abstract}

Keywords: Multi-objective / irrigation / pareto set / evolutionary algorithm

\section{Introduction}

Water is an essential natural resource because the lives of living organisms depend on it. Agriculture, Industries, and Domestic activities are the major water users. Under pressure from population explosion, urbanization, extravagant lifestyles, climate change, intensive agriculture and industrialization, water are fast becoming a scarce resource. This is evident from the fact that lack of water to meet the daily requirement is a reality for one in three people globally [1].

Demand for food due to population growth and climatic change makes agricultural sector the most water consumer in the world. Agricultural activities consume 70\%, while industrial and domestic activities consume $30 \%$ of world water. Water use management is an important field for the assurance of effective and sustainability of food supply [2].

The water management challenge can be addressed in a different way, currently, global optimization techniques appeared to be promising methods for optimizing water consumption strategies. The aim of these techniques in irrigation planning and crop production is to attain maximum crops productivity under deficit water supply within an irrigated land [3]. There are several optimization

\footnotetext{
* e-mail: fanueli@nm-aist.ac.tz
}

techniques used worldwide to analyze the optimization problem with several objectives as discussed in [4].

Several problems in engineering, industry, economy and many other fields, including water management involve the optimization of several objectives simultaneously. The objectives are defined in incomparable units, and express some degree of conflict among them. Proper irrigation planning involves optimization of several conflicting objectives [5-9]. Among the optimization techniques employed to solve multi-objective irrigation problem are Multi-Objective Evolutionary Algorithms (MOEA) and are the central focus of this review.

The review is based on the published articles from 1995 to 2016 which focused on formulating the model for irrigation water allocation problem and used MOEA to analyze the model formulated. The articles were collected from the journals with the scope of water resource management, Mathematical optimization, numerical analysis, and multi-objective evolutionary algorithms. To find papers related to this work, the following keywords were used on bibliographical databases and search engines, such as Science Direct and Google Scholar: mathematical models for irrigation optimization, multi-objective evolutionary algorithms, irrigation, optimization, genetic algorithms, non-sorting genetic algorithms and differential evolution. Moreover, the bibliographical references of the articles have served as a continuous search reference. 


\section{Evolutionary algorithm}

Evolutionary Algorithms (EA's) are a set of heuristic methods that are inspired by the organic selection mechanisms such as mutation, recombination and natural selection in finding global solutions to the optimization problem. These techniques are employed to solve optimization problem with conflicting objectives after researchers identified several limitations that traditional mathematical programming methods encountered in solving optimization problem with several objectives. According to [10] and [11], traditional mathematical programming techniques have the following drawbacks: single run does not offer several elements of Pareto set; understanding of the domain knowledge of the problem is required, and some of the mathematical programming techniques are very sensitive to the shape or continuity of the Pareto front [12].

Therefore Multi-Objective Evolutionary Algorithms are techniques that are suitable to be used because the objective functions are simultaneously solved, this allows approximation of the entire Pareto optimal set in a single run of the algorithm rather than performing a series of distinct runs as in the case of the traditional mathematical programming techniques [10].

\section{Multi-objective evolutionary algorithms in irrigation water allocation optimization}

The multi-objective problem is the kind of problem which consists many objective functions that are to be either maximized or minimized. As in single objective problem, the multi-objective problem may involve some equality, inequality or bound constraints which any feasible solutions including optimal solutions must satisfy. The general form of a Multi-Objective Problem (MOP) can be stated as [4,13]:

$$
\max / \min f_{m}(x), m=1,2,3 \ldots, M ;
$$

subject to:

$$
\begin{gathered}
g_{j}(x) \geq 0, j=1,2,3 \ldots, J ; \\
h_{k}(x)=0, k=1,2,3 \ldots, K ; \\
x_{i}^{L} \leq x_{i} \leq x_{i}^{U}, i=1,2,3 \ldots, n .
\end{gathered}
$$

In the viewpoint of optimizing multi-objective problem, when all objective functions are important, finding the optimal solution cannot be done to only one objective function. Different solutions may produce contradictory outcomes among the objectives, there is no possibility of choosing the solution that gives the optimal value of only one objective function. This complexity suggests the important steps of multi-objective optimization [13]:

- find a set of solutions which lie on the Pareto-optimal front, and

- find a set of solutions which are varied enough to represent the complete range of the Pareto-optimal front.

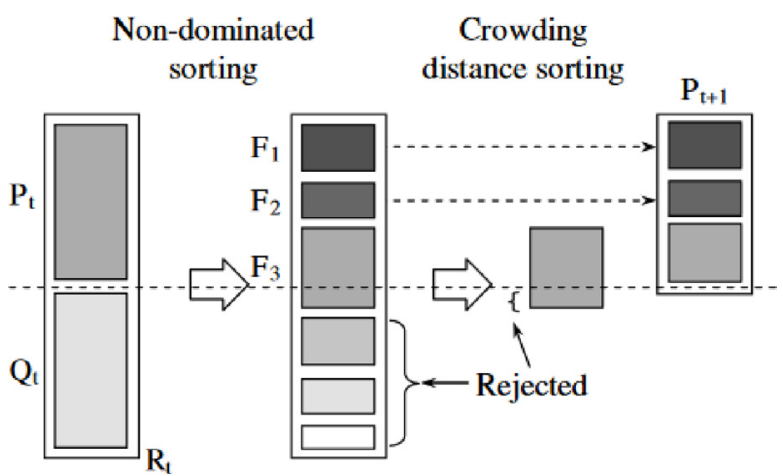

Fig. 1. NSGA-II Procedures (Adapted from [18]).

MOEA follow the steps in finding the optimal solutions of the MOP. This paper focused on the application of the three MOEA; Genetic Algorithm (GA), Non-Sorting Genetic Algorithm-II (NSGA-II) and Multi-Objective Differential Evolution (MODE). The choice of these methods is based on the number articles published, computational performance, their similarities on using Pareto technique and trend of researchers to apply MOEA to solve multi-objective problem.

\subsection{Genetic algorithm (GA)}

This is an EA method that solve both constrained and unconstrained optimization problem based on a natural selection process that mimics biological evolution [14]. The method consists of a structure in which the rank of a certain individual matches to the number of individuals in the current population by which it is dominated, randomly selects individuals at each step from the present population and treat them as parents to produce the children for the next generation. Over the sequential generations, the population advances toward the optimal solution $[4,15]$.

The assignment is performed in the following way as proposed by [14]: If an individual $x_{\mathrm{i}}$ at generation $t$ which is dominated by $p_{i}^{(t)}$ individuals in the current situation then the new position of the individuals' rank is given by $\operatorname{rank}\left(x_{i}, t\right)=1+p_{i}^{(t)}$.

\subsection{Non-dominating sorting genetic algorithm-II (NSGA-II)}

NSGA is EA method proposed by [16] to solve the multiobjective optimization problem based on various sheets of classification of individuals. The population is ranked based on non-domination before selection process.

NSGA-II is the improved version of NSGA proposed by $[17,18]$. Figure 1 below shows the NSGA-II procedures in finding the best solutions. It starts with building a population of rival individuals, ranks, and sorts according to its non-domination level, then it creates a new offspring pool by using Evolutionary Operators, and finally combines the parents and offspring before splitting the new combined pool into fronts.

$P_{t}$ is the parents' population and $Q_{t}$ is the offspring population at generation $t$. $F_{1}$ are the best solutions from 


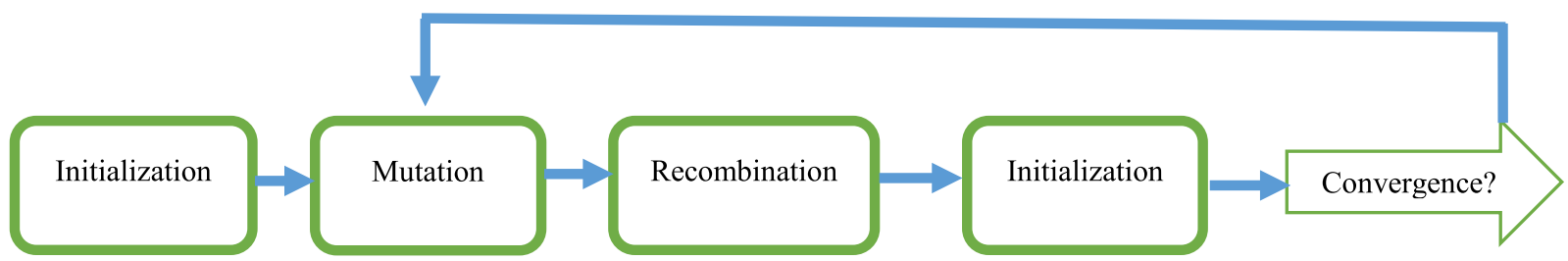

Fig. 2. Differential Evolution Procedure.

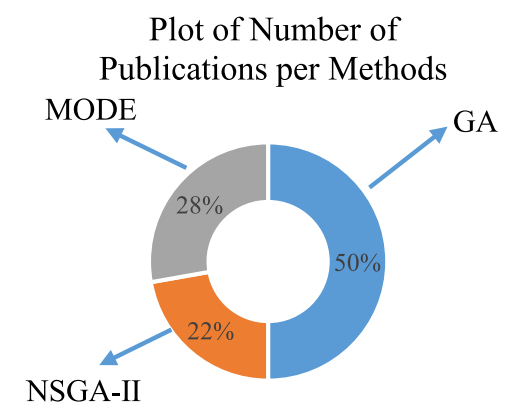

Fig. 3. Evaluation of Publications per Method.

the combined populations (parents and offspring); $F_{2}$ are the second-best solutions and so on.

\subsection{Multi-objective differential evolution (MODE)}

This is the kind of evolutionary algorithm proposed by [19] for solving multi-objective problem over a continuous domain. It is as simple tool to be employed but powerful algorithm with many successful applications [20]. Comparable to other evolutionary algorithms, the components of MODE approach are; reproduction, evaluation, and selection.

For every individual in the parent population, MODE uses the following reproduction operator to create the offspring [21]:

$$
p_{i}^{\prime}=\gamma p_{b}+(1-\gamma) \cdot p_{i}+F \cdot \sum_{k=1}^{K}\left(P_{i}-P_{i}\right)
$$

where $p_{b}$ is the best individual in the parent population, $\gamma$ represents voracity of the operator, and $K$ is the number of perturbation vectors, $F$ is the scale factor of the perturbation, $p_{a}^{k}$ and $p_{b}^{k}$ are randomly selected mutually distinct individual pairs in the parent population, and $p_{i}^{\prime}$ is the generated offspring; $\gamma, K$, and $F$ are algorithm's parameters.

Figures 3 and 4 visualizes 18 publications which applied multi-objective evolutionary algorithms (Genetic Algorithms, Non-Sorting Genetic Algorithms, and MultiObjective Differential Algorithms) to find the solutions of water allocation multi-objective problem from 1995 to 2016. The figures indicate that, Genetic Algorithm has been widely exploited and from 2007 to 2016 there are more publications used Multi-Objective Differential Evolution to solve water management multi-objective problem.

Formulation of the multi-objective optimization model for the irrigation water allocation with the aim of maximizing water productivity has been a research area

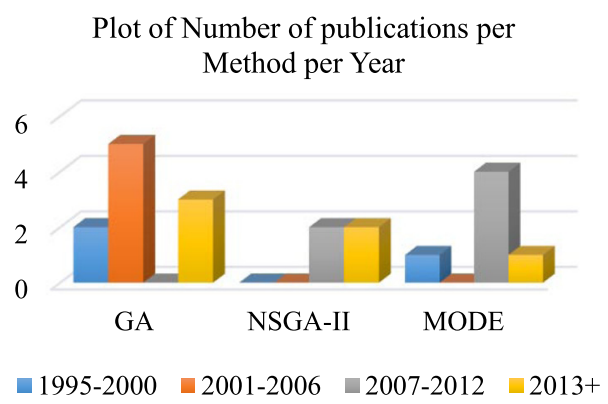

Fig. 4. Evaluation of Publications per Method per Year.

of interest to many researchers. For example [11] employed Multi-Objective Genetic Algorithm (MOGA) to determine the optimal reservoir operation. Elitism mechanism and the crowded distance operator were used to obtain several Pareto set which were very attractive and efficient within single run, from which decision maker can take appropriate decisions at different levels.

Ref. [22] applied GA to develop effective cropping patterns for maximizing benefits for an irrigation project in India. The results obtained from the GA model were related with those obtained from the Linear Programming model, the comparison show that GA is an effective optimization technique for irrigation planning and can be used for more multifaceted systems involving non-linear optimization.

Ref. [23] did a study which focused on a decision support system for irrigation project planning using GA, the problem focused on optimization of net benefit, crop area percentages, standard deviation, and water demand. Three methodologies were used and the results were compared. They claimed that Genetic Algorithm and Simple Algorithm methods are preferred over the iterative improvement to help irrigation managers or governmental agencies to reach irrigation project planning decisions.

Ref. [24] examined the development of " $A L L_{-}$$W A T E R$ " as the multi-objective tool for optimizing water demand and the unit costs incurred during crop production. MOGA and the PARETO optimality idea were used to resolve the formulated problem and computing the decision variables of the problem. The model demonstrated the efficiency of the methodology developed, from which a set of 72 the optimal solutions and Pareto front were obtained in a single run within a short computational time. The results show that, the developed tool is efficient and stretchy for water resource management optimization for all types of water resources.

Ref. [25] applied GA to solve a non-linear programming optimization model with an integrated soil/water balance 


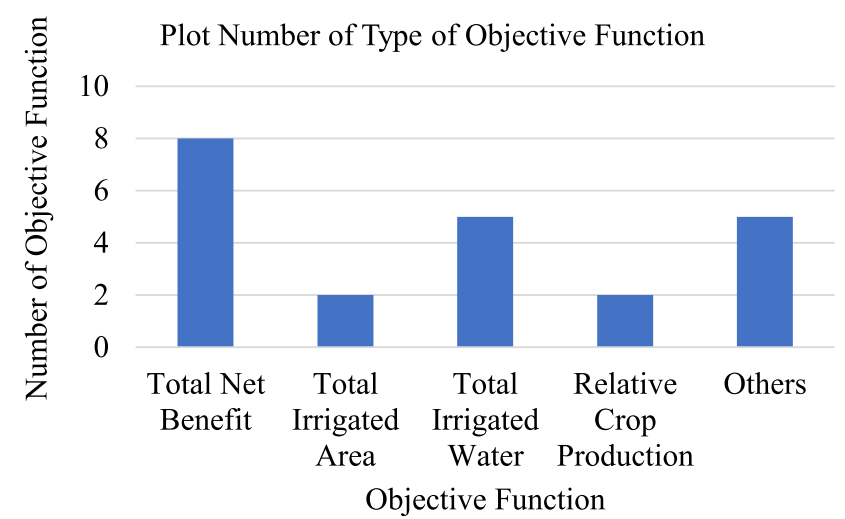

Fig. 5. Objective Functions of the articles published from the year 1995 to 2016 .

to identify the optimal reservoir release approaches and the optimal cropping pattern. The model shows suitable performance as the model tends to converge to a maximum after 200 iterations, total farm benefit and the total cultivated area under deficit irrigation were larger than those under full irrigation.

Ref. [26] propose a model for off-farming irrigation focused on the optimization of the number of orders that are planned to be distributed at the requested time and variations in the channel flow rate are to be optimized. The results show that GA techniques can identify good irrigation order schedules.

Ref. [27] did a comparison of the results obtained from Quadratic Programming (QP) and GA for the water allocation in an irrigation system problem. GA produced the same solutions as QP, meanwhile, it was found to be sensitive to string length and has difficulty in meeting closed nodal water balance constraints, and the claim is GA approach offered no pro over the QP for the water distribution problem.

Apart from the above mentioned studies, there is a number of studies that describe efficiency and strength of using used Genetic Algorithm as an optimization tool to provide considerable solutions for irrigation water allocation management and is an optimization tool which is more exploited than other evolutionary tools, meanwhile, its performance is highly dependent on an appropriate selection of the sharing factor [10].

The second EA's in this review is NSGA-II, this is the kind of Genetic Algorithm but with a slight difference on how selection operator works. The crossover and mutation operators are the same $[16,28]$. The advantage of using NSGA-II over other evolutionary algorithms is that NSGA-II is the method based on operator selection. The approach has not been comprehensively tested yet like MOGA, there is a room for researchers to invest on NSGAII because looks promising.

Ref. [29] conducted a study on water allocation to agricultural areas from three sources of water; rainfall, river and ground water by using a NSGA-II to obtain the Pareto front. The problem involves maximization of two objective functions (Net benefit and comparative water use efficiency) with water and land as decision variables. The irrigation schedule was applied in the field located at
Baghmalek plain in Iran for experimental, whereby the model did not propose deficit irrigation for melon and tomato during the middle stage and suggest that NSGA-II improves precision in irrigation scheduling.

The study carried by [30] proposed a model to manage the underground and surface water resources' conjunctive use. The problems were to optimize the minimum reliability of the system and the costs of water supply, aquifer renovation and violation of the reservoir capacity in operation and allocation priority. The NSGA-II method was used to present the optimal Pareto front between the objectives. The results obtained from NSGA-II were compared with the results from Sequential Genetic Algorithms (SGA). The comparison showed that the NSGA-II model can significantly reduce the computation problem of the conjunctive use models over the use of SGA optimization model.

Ref. [31] used the NSGA-II to examine the operations of a multi-reservoir system in Taiwan. The analysis showed that the NSGA-II method gave improved operating rule curves than the original operating curves and reduce the Shortage Indexes (SI) of the reservoirs effectively. SI was used to describe the state of the reservoir, the smaller the SI the much surplus water and the higher SI the serious water shortage problem. The results described NSGA-II as convincing and vital evolutionary algorithm to recognize dual operation methodologies that will address the proper use of resources for future use.

Another EA method used in solving irrigation water allocation models is the recent method in the family of evolutionary algorithm methods which is the combination of non-dominated sorting and Pareto-optimality principles. The method is called MODE. According to [32], MODE technique is superior in performance to NSGA-II and is an appropriate method for problems having interreliant relationships between the decision variables in problems of reservoir operation.

Ref. [33] suggested approaches of MODE to solve the multi-objective problem of crop planning with multiple constraints in a farmland. The results showed that MODE algorithm can be used solve crop planning problem effectively, especially in water scarce areas and is operative and dynamic multi-objective optimization algorithm for solving the multi-objective model.

Ref. [34] conducted a research on the application of MODE and Differential Evolution (DE) for Irrigation Planning. The problem was to optimize net benefits, agricultural production and labor employment where by four strategies of DE were employed, namely, DE/rand/1/ bin, DE/rand/1/exp, DE/best/1/bin and DE/best/1/exp.

Comparison analysis showed that $\mathrm{DE} / \mathrm{rand} / 1 / \mathrm{bin}$ generated better Pareto-optimal curve in MODE scenario and $\mathrm{DE} /$ best $/ 1 /$ bin performed better in $\mathrm{DE}$ scenario. Hence MODE with DE/rand/1/bin is efficient multiobjective solver.

Ref. [35] employed MODE to solve the problem of optimizing water allocation optimally through the description of economic, social and Eco-environmental benefits. The model tested by using the real data from NingxiaChina, the results show that MODE is rational and feasible algorithm in the application of water distribution, and the 
model can be employed to enhance the utilization level of water resources, inspire environmental conservation and improve the wide-ranging operation benefit of water resources. Other studies suggest that MODE is a simple and powerful evolutionary optimization technique which is faster than other evolutionary techniques and robust in finding the global optimum [19] and can be employed for efficient planning of any irrigation system with a suitable adjustments [36].

The following are the interpretations from the reviewed literatures: (1) Evolutionary Algorithms are powerful and efficient methods to obtain quality solutions of the Multiobjective problem in a single run (2) Most of the studies focus on maximization of net benefit/net income without consideration of minimizing the variable costs incurred during crop production (3) Few research works in the field of irrigation planning in multi-objective evolutionary algorithm framework focused on extensive sensitivity analysis of the model parameters.

\section{Discussion}

The aspects for discussion can be examined from different scenarios which are of interest in model formulation and implementation, for this paper, two scenarios are considered as vital in multi-objective optimization problem. The first is, modeling or problem formulation (objective functions, decision variables, parameters, and constraints); and the second scenario focused on the solution methods (GA, NSGA-II and MODE with respect to this review).

\subsection{Objective functions, decision variables, parameters and constraints}

In this review one objective function was observed in most publications, namely maximizing net benefit/farm income objectives. Out of more than 22 reviewed objective functions (in single or multi-objective problems), 8 (36.4\%) publications have the objective functions related to maximizing total net benefit from the irrigation system. Figure 4 visualize the distribution of objective functions per publications.

Meanwhile, there are a considerable number of parameters and constraints, some of the parameters are: Evapotranspiration, maximum and minimum temperature, mean wind speed, soil moisture, root depth, coefficient of albedo and average rainfall as in [32,37-39]. The model consists of the constraints that ensures efficient use of available water and land, soil moisture balance, reservoir continuity, scheduling constraint and environmental flow [38,40-44].

\subsection{Solution methods}

In this paper, we reviewed articles which used MOGA, NSGA and MODE as the solution methods to obtain Pareto optimal set of a multi-objective irrigation problem.

As other stochastic search strategies, MOEA does not offer a true Pareto optimal set of solutions, instead, the techniques aim to offer a good approximation of entire Pareto set in a realistic computational time [45]. MOGA and NSGA-II both use Pareto ranking techniques where by the computational performance is extremely dependent on the proper selection of the sharing factor. For a constrained Multi-Objective Optimization Problem stated as in equations (1)-(4) above, the theoretical Pareto-optimality conditions proposed by $[45,46]$ are used to obtain the best solutions for the problem:

For $x^{*}$ to be Pareto optimal there must exist vectors $\lambda \geq 0$ and $\mu \geq 0$ (where $\lambda \in \mathbb{R}^{M}, \mu \in \mathbb{R}^{J}$ and $\lambda, \mu \neq 0$ ) such that the following conditions are true:

$$
\sum_{m=1}^{\mathrm{M}} \lambda_{m} \nabla f_{m}\left(x^{*}\right)-\sum_{j=1}^{J} \mu_{j} \nabla g_{j}\left(x^{*}\right)
$$

and

$$
\mu_{j} g_{j}\left(x^{*}\right)=0 \text { for all } j=1,2, \ldots, J
$$

\section{Conclusion and future research agenda}

From this review, it can be generalized that many researchers worldwide have formulated, introduced and employed various EA's to solve irrigation problem from a single objective to multi-objective problem. The results obtained showed that EA's are powerful and reliable methods to estimate the Pareto-optimal solutions. Particularly, GA seems to be extensively exploited, whereby, there are numerous works that applied GA, with a number of free downloadable compilation codes.

The review also shows that Multi-objective differential evolution is the recent algorithm from the family of EA's which is simple and powerful than any EA methods, meanwhile, the published comparative studies mostly remain qualitatively. Therefore, with a reference to the scope of the paper, there is a need to answer the following questions:

- which EA are suitable to which class of problem?

- what is the sufficient and acceptable baseline for quantitative performance metrics for multi-objective optimizers?

Also, the review indicates that optimizing the net benefit from the total irrigated area has been of more interest to many researchers, on the other hand, optimization of the total variable costs related to irrigation practice and crops production and the benefits that can be generated from the farm byproducts after harvesting have not been considered. Nevertheless, in an economic point of view, optimizing the total net benefit, consideration of the costs bared during the process of production is an important aspect.

\section{Conflict of interests}

The authors declare that there is no conflict of interests.

\section{References}

1. O. Olofintoy, J. Adeyemo, F. Otieno, Evolutionary algorithms and water resources optimization, in EVOLVE - A bridge between probability, set oriented numerics, and evolutionary computation II (2013) pp. 491-506 
2. A. Ikudayisi, J. Adeyemo, Environ. Econ. 6, 200-205 (2015)

3. N. Schütze, T. Wöhling, M. De Paly, G. Schmitz, Global optimization of deficit irrigation systems using evolutionary algorithms, in Proceedings of Denmark, Proceedings of the XVI International Conference on Computational Systems (2006)

4. C.A.C. Coello, G. Pulido, Evolutionary Multi-Criterion Optimization, in A Micro-Genetic Algorithm for Multiobjective Optimization (2001) Vol. 1993, pp. 126-140

5. Y. Han, Y-F. Huang, G-Q. Wang, I. Maqsood, Water Resour. Manag. 25, 449-463 (2011)

6. W.R. Godoy, A.F. Barton, I. Victoria, A Procedure for Formulation of Multi-Objective Optimisation Problems in Complex Water Resources Systems, in 19th International Congress on Modelling and Simulation (2011), pp. 12-16

7. R. Lalehzari, S. Boroomand Nasab, H. Moazed, A. Haghighi, J. Irrig. Drain. Eng. 142, 5015008 (2016)

8. M. Habibi Davijani, M.E. Banihabib, A. Nadjafzadeh Anvar, S.R. Hashemi, Water Resour. Manag. 30, 927-946 (2016)

9. E. Xevi, S. Khan, J. Environ. Manage. [Internet] 77, 269-277 (2005)

10. L. Antonio, C.A.C. Coello, Z. Mart, Knowl. Inf. Syst. 1, 269 308 (1999)

11. M.J. Reddy, D.N. Kumar, Water Resour. Manag. 20, 861878 (2006)

12. L. Antonio, C.A.C. Coello, Z. Mart, An introduction to multiobjective (2009), pp. 1-26

13. K. Deb, Multi-objective optimisation using evolutionary algorithms: an introduction, in Multi-objective evolutionary optimisation for product design and manufacturing (2011), pp. 3-34

14. C.M. Fonseca, P.J. Fleming, Genetic Algorithms for Multiobjective Optimization: Formulation, Discussion and Generalization, in Icga (1993), pp. 416-423

15. MathWorks, MathWorks.com. Genetic Algorithm - MATLAB Simulink [Internet] (2017), p. 1

16. N. Srinivas, K. Deb, Evol. Comput. 2, 221-248 (1994)

17. K. Deb, S. Agrawal, A. Pratap, T. Meyarivan, A Fast Elitist Non-dominated Sorting Genetic Algorithm for Multi-objective Optimization: NSGA-II, in Lecture notes in ... (2000) pp. 849-858

18. K. Deb, A. Pratap, S. Agarwal, T. Meyarivan, IEEE Trans. Evol. Comput. 6, 182-197 (2002)

19. R. Storn, K. Price, J. Glob. Optim. 11, 341-359 (1997)

20. T. Robič, B. Filipič, DEMO: Differential Evolution for Multiobjective Optimization, in Evolutionary Multi-Criterion Optimization (2005) pp. 520-533

21. F. Xue, A.C. Sanderson, R.J. Graves, Pareto-based multiobjective differential evolution, in The 2003 Congress on Evolutionary Computation, CEC '03 (2003) pp. 862-869

22. K. Srinivasa Raju, D. Nagesh Kumar, Water Resour. Manag. 18, 163-176 (2004)
23. S. Kuo, G.P. Merkley, C. Liu, Agric. Water Manag. 45, 243$266(2000)$

24. I. Nouiri, Water Resour. Manag. 28, 2885-2901 (2014)

25. S.K. Sadati, S. Speelman, M. Sabouhi, M. Gitizadeh, B. Ghahraman, Water (Switzerland) 6, 3068-3084 (2014)

26. J.B. Nixon, G.C. Dandy, A.R. Simpson, J. Hydroinf. 3, 11-22 (2001)

27. R. Wardlaw, K. Bhaktikul, Irrig. Drain. 50, 159-170 (2001)

28. X. Jin, J. Zhang, J. Gao, W. Wu, J. Zhejiang Univ. A 9, 391400 (2008)

29. R. Lalehzari, S. Boroomand Nasab, H. Moazed, A. Haghighi, J. Irrig. Drain Eng. 142, 5015008 (2016)

30. M.M.R. Tabari, J. Soltani, Water Resour. Manag. 27, 37-53 (2013)

31. L-C. Chang, F. Chang, J. Hydrol. 377, 12-20 (2009)

32. M.J. Reddy, D.N. Kumar, Irrig. Sci. 26, 177-190 (2008)

33. J. Adeyemo, F. Otieno, Agric. Water Manag. 97, 848-56 (2010)

34. P. Gupta, A. Vasan, K.S. Raju, Multiobjective Differential Evolution and Differential Evolution for Irrigation Planning, in World Environmental and Water Resources Congress (2009), pp. 5022-5029

35. K.P. Feng, J.C. Tian, Appl. Mech. Mater. 278-280, 12711274 (2013)

36. A. Vasan, K.S. Raju, Water Resour. Manag. 21, 1393-1407 (2007)

37. J. Adeyemo, F. Otieno, Application of multi-objective differential evolution algorithm (MDEA) to irrigation planning, in World Environmental and Water Resources Congress (2009), pp. 4689-4698

38. M.S. Babel, A. Gupta Das, D.K. Nayak, Water Resour. Manag. 19, 693-712 (2005)

39. K.S. Raju, A. Vasan, P. Gupta, K. Ganesan, H. Mathur, ISH J. Hydraul. Eng. [Internet] 18, 54-64 (2012)

40. H. Noory, A.M. Liaghat, M. Parsinejad, O.B. Haddad, J. Irrig. Drain. Eng. [Internet] 138, 437-444 (2012)

41. S. Yakowitz, Water Resour. Res. 18, 673-696 (1982)

42. D.C. McKinney, X. Cai, Multiobjective optimization model for water allocation in the Aral Sea basin, in Conference paper, American Institute of Hydrology-CIS (1996)

43. B. Ghahraman, A-R. Sepaskhah, Irrig. Drain. 53, 39-54 (2004)

44. R. Roozbahani, S. Schreider, B. Abbasi, Multi-objective decision making for basin water allocation, in 20th International Congress on Modelling and Simulation (2013), pp. 1-6

45. D.A. Veldhuizen Van, G.B. Lamont, Evolutionary Computation and Convergence to a Pareto Front, in Late breaking papers at the genetic programming1998 conference (1998), pp. $221-228$

46. K. Deb, K. Sindhya, J. Hakanen, Multi-Objective Optimization, in Decision Sciences [Internet] (2016), pp. 145-184

Cite this article as: Ibrahim Mwita Fanuel, Allen Mushi, Damian Kajunguri, Irrigation water allocation optimization using multiobjective evolutionary algorithm (MOEA) - a review, Int. J. Simul. Multidisci. Des. Optim. 9, A3 (2018) 